and as the phenomenon passed onwards the light grew in brightness until it equalled the lustre of the electric arc, and has been compared to the glow of a great electric search-light. The emitted light lasted at least 30 seconds. Apparently the path was from the north-west. Two minutes after disappearance three detonations were heard, the last being of exceptional violence, shaking buildings, and causing the earth to vibrate. Here at Henwick, the head of the meteor, though visible at other places, was invisible, but a magnificent long luminous trail was apparent. At Hallow, hence three miles north, and at Clifton-on-Teme, hence twelve miles north-west, the light was seen, and the effects of the terrific explosion were experienced. At the former place the crockery-ware was jarred off the shelves of cottages. A loud rumbling noise was also heard, some persons describing it as like the prolonged roar of distant thunder. At the Wych Malvern, slates were displaced from house-roofs. A gentleman who observed the meteor at Mold, North Wales, says that, if anything, it appeared to him to be larger than the moon. The colour 'was blue in the centre, and had yellow fire round the edges.' No explosion was heard there. The meteor, it is supposed, broke up near Clifton-on-Teme, but no trace of its débris has hitherto been found. At Droitwich, hence seven miles north-east, it was thought that the Evesham gas works, twelve miles away, had blown up. At Pershore the head of the meteor was seen, and its bursting, which it is said was accompanied by the flashing of a dull red light, was witnessed. At Malvern, eight miles westward, the terrific effects of the occurrence were apparent. Here there is no previous record of a meteor on so grand, prolonged, and terrific a scale."

Several letters describing the meteor have appeared in the Times. Mr. W. H. Lloyd observed the phenomenon from the top of the Cotswolds, about half a mile north of Minchin Hampton. He saw a ball of fire pass rapidly from north to south, and disappear in one or two seconds. About a minute or a minute and a half afterwards a series of explosions was heard. A de-

tonating sound was also heard at Cheltenham, but at some other places no peculiar sound was noticed. A loud rumbling noise like an explosion was heard near Ross, Herefordshire, and ascribed to an earthquake shock. Mr. J. G. Wood remarks in yesterday's Times that there is possibly a connection between earthquakes and meteoric phenomena. He points out that the North Devon disturbance of January 23 (see p. 320) was followed by the meteor of January 25 , and that both an earthquake and a bright light was observed at Ross, though the observer did not actuaily see a meteor. The light of the meteor is variously stated, but the majority of observers describe it as intensely brigbt and bluish, similar to the light of the electric arc. Mr. J. D. La Touche, writing from Stokesay Vicarage, Shropshire, says that the phenomenon continued for certainly more than half a minute; but at Brixworth. Northampton, the duration is said to have been about seven or eight seconds. All agree, however, that the meteor was of a brilliancy so great that the whole sky was illuminated, and Venus and Jupiter paled into insignificance before it.

\section{ON A METEORITE FROM GILGOIN STATION:}

I T will be remembered that at the June ( 1889 ) meeting of the

Society I exhibited a meteor weighing $67 \frac{1}{2} \mathrm{lbs}$. sent to me by Mr. J. F. Yeomons, of Gilgoin Station, situated forty miles towards east south-east from Brewarrina. (This meteorite is the left-hand one shown in the accompanying figure.) It had been long exposed to the weather, and the chemical action of air and rain had broken up the surface of it to such an extent that pieces fell off each time it was handled.

On February 8, 1893, Mr. Yeomons again wrote to me and said :- "We have in our possession an aerolite, found, a short

1 Read at the Royal Society, Sydney, November I, 1893.

NO. I 266, vOL. 49] time since, about two miles south of the one sent you some time ago. I can have it sent to you by train from Byrock." Various delays occurred, and I did not get it until September 5. The meteor had been very carefully packed, and had not suffered much loss on the journey, although, like the previous one from this locality, it is much cracked, and many parts of the surface are ready to crumble away. All the parts together weigh $74 \frac{1}{2} \mathrm{lbs}$, and its specific gravity as a whole is 3.757 . The No. I Gilgoin meteor weighs $67 \frac{1}{2} \mathrm{lbs}$. and its specific gravity is 3.857 . They are so much alike that it strengthens the probability arising from exterral similarity and nearness of the localities in which they were found that they are parts of one much larger. It is but right, however, to add that if so, they must have travelled through the atmosphere together a sufficient distance to cause the usual melted surface, which, although in parts lost by subsequent slow effect of oxidation, is yet too extensive to admit the alternative that they divided as they fell.

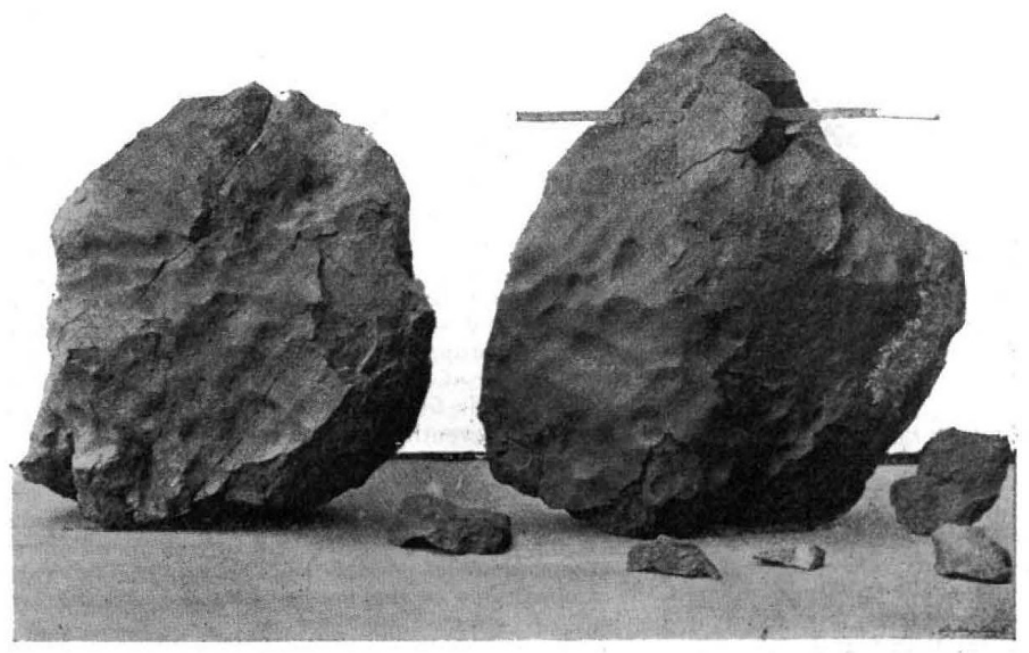

This recently-found No. 2 Gilgoin meteor is, roughly, double convex, and measures 7 inches through the thickest part, and $14 \times 15$ inches diameter. The surface has been melted, but is not so smooth and glossy as others I have seen; when a part of it which has not been oxidised is broken, it is dark grey in colour, and shows a great abundance of fine bright, white metallic particles. The rule is laid in a space left by some pieces missing. The meteorite has not yet been analysed, but I hope Prof. Liversidge will undertake that work.

\section{H. C. Russell.}

\section{MODERN MATHEMATICAL THOUGHT ${ }^{1}$}

$\mathrm{NE}$ who, like myself, is not a mathematician in the modern sense naturally feels that some apology is due for accepting the invitation with which this society has honoured me, to address it on a mathematical subject. Possibly an adequate apology may be found in the reflection that one who has not gone deeply into any of the contemporaneous problems of mathematics, but who, as a student, has had a sufficient fondness for the subject to keep himself informed of the general course of thought in it, may be able to take such a general review as is appropriate to the present occasion. I shall there. fore ask your consideration of some comparisons between the mode of thinking on mathematical subjects at the present time, and those methods which have come down to us from the past, with a view of pointing out in what direction progress lies, and what is the significance of mathematical investigation at the present day.

Among the miscellaneous reading of my youth was a history of modern Europe, which concluded with a general survey and attempted forecast of progress in arts, science, and literature. So far as I can judge, this work was written about the time of

1 Address delivered before the New York Mathematical Society at the annual meeting, December 28, 1893, by Prof. Simon Newcomb. 
Euler or Lagrange. On the subject of mathematics the writer's conclusion was that fruitful investigation seemed at an end, and that there was little prospect of brilliant discoveries in the future. To us, a century later, this judgment might seem to illustrate the danger of prophesying, and lead us to look upon the author as one who must have been too prone to hasty conclusions. I am not sure that careful analysis would not show the author's view to be less rash than it may now appear. May we not say that in the special direction and along the special lines which mathematical research was following a century ago no very brilliant discoveries have been made? Can we really say that Euler's field of work has been greatly widened since his time? Of the great problems which baffled the skill of the ancient geometers, including the quadrature of the circle, the duplication of the cube, and the trisection of the angle, we have not solved one. Our only advance in treating them has been to show that they are insoluble. To the problem of three bodies we have not added one of the integrals necessary to the complete solution. Our elementary integral calculus is two centuries old. For the general equation of the fifth degree we have only shown that no solution exists. We should, doubtless, solve many of the problems which the Bernoullis and their contemporaries amused themselves by putting to each other, rather better than they did; but, after all, could we get any solution which was beyond their powers? I speak with some diffidence on such a point as this; but it seems to me that progress has been made by going back to elementary principles, and starting out to survey the whole field of mathematical in vestigation from a higher plane than that on which our predecessors stood, rather than by continuing on their lines.

We may illustrate this passage to new modes of thought by comparing Euclid's doctrine of ratio and proportion with our own. No one questions the beauty or rigour of the process by which Euclid developed this doctrine in his fifth book, and applied it to the theory of numbers in his seventh book. But can we help pitying our forefathers who had to learn the complex propositions and ponderous demonstrations of the fifth book, all the processes and results of which we could now write on a single sheet of paper? As a mental discipline the study was excellent; but it seems hardly possible that one could have remembered the propositions or the methods of demonstrating them if he had no other knowledge of them than that derived from the work itself. When we carefully examine these propositions, we find that while Euclid recognised the fact that one of two ratios might be greater than, equal to, or less than another, yet he never regarded them as mere quantities which could be treated as such. From his standpoint a ratio was always a relation, and a relation cannot exist without two terms.

In pointing out this complexity of Euclid's doctrine, I must not be taken to endorse the very loose way in which the doctrine in question is usually treated in our modern textbooks. What we should aim at is to replace Euclid's methods by those which pertain to modern mathematics. At the present time we conceive that a relation between any two concepts of the same kind may always be reduced to a single term by substituting for it an operator whose function it is to change one of these concepts into the other. In the case of the relation between two lines, considered simply as one dimensional quantities, which relation is called a ratio, we regard the ratio as a numerical factor or multiple, which, operating on one line, changes it into the other. For example, that relation which Euclid would have expressed by saying that two lines were to each other as 5 to 2 , or that twice one line was equal to five times the other, we should now express by saying that if we multiplied one of the lines by two and one half, we should produce the other. This might seem to be simple difference of words, but it is much more. It is a simplification of ideas; a substitution of one conception for two. Euclid needed two terms to express a relation; we need but one.

But this is not the only simplification. A peculiarity of our modern mathematics is that operators themselves are regarded as independent objects of reasoning; susceptible of becoming operands, without specification of their particular qualities as operators. Thus, instead of considering the ratio which $I$ have just mentioned as an operation of multiplying a line by two and one half, we finally reduce it to the simple quantity two and one half, which we may conceive to remain inert until we bring it into activity as a multiplier. It thus assumes a concrete form, capable of being carried about in thought, and operated upon as if it were a single thing.

This example may afford us a starting-point for a farther illustration of the way in which we have broadened the conceptions which lie at the basis of mathematical thought. Let us reflect upon the relation between a straight line going out from a certain point, and another line of equal length going out from the same point at right angles to the first. Had this relation been presented to Euclid as a subject for study, he would pro. bably have replied that though much simpler than those he was studying, he could see nothing fruitful in it, and would have drawn no conclusions from it. But if we trace up the thought we shall find a wide field before us, embracing the first conception of groups, and with it an important part of our modern mathematics. In accordance with the principle already set forth, we replace the relation between these two lines by an operator which will change the first into the second. We define this operator by saying that its function is to turn a line through a right angle in a fixed plane containing the line. This definition permits of the operator in question being applied to any line in the plane. Then let us apply it twice in succession to the same line. The result will be a line pointing in the opposite direction from the original one. A third operation will bring it again to a right angle on the opposite side from the second position; and a fourth will restore the line to its original position, the result being to carry it through a complete circle. If we now consider the operations which would have been equivalent to these one, two, three, and four revolutions through a right angle as four separate operators, we see that their results will be either to leave the line in its original position, or to move it into one of three definite positions. If we then repeat one of these four operations as often as we please, or in any order we please, we shall only bring the line to one of the four positions in question. We thus have a group of the fourth order, possessing the property that the repetition of any two operations of the group is equivalent to some single operation of it.

I scarcely need call attention to the familiar homology between these operations and successive multiplications by the imaginary unit $1-\mathbf{I}$. This last concept, considered as a multiplier, has the same properties as our rotating operator. Repeated twice, it changes the sign or direction of the quantity on which it operates; repeated four times, it restores it to its original value. Let us extend this idea a little. Instead of taking two lines at right angles to each other, let us consider two which form an angle of $40^{\circ}$. As already remarked, this relation is homologous with an operator which will turn a single line through that angle. If we continually rep eat this operation, we shall bring the line into thirty-five different positions, the thirty-sixth position being identical with the original one. Thus we should have thirty-six positions in all, expressed by that number of lines radiating from a single centre, and making angles of $10^{\circ}$ with each other. Now let us imagine thirty-six operators whose function it is to turn a line, no matter what, successively through an arc of $10^{\circ}, 20^{\circ}$, $30^{\circ}$, \&c. up to $360^{\circ}$, the last being equivalent to an operator which simply does nothing. These thirty-six operators will form a group which we know to be strictly homologous with multiplication by the thirty-six expressions

$$
e^{i \phi}, e^{2 i \phi}, \epsilon^{3 i \phi}, \ldots e^{36 i \phi}=e^{\circ}=\mathbf{I},
$$

where $\phi$ is the arc of $10^{\circ}$ in circular measure.

So far we have only considered operations formed by the continual repetition of a single one; in the language of the subject, all our groups are constructed from powers of a single operator. Now let us extend our process by substituting a cube for our straight line. Through this cube we have an axis parallel to four of its plane sides. By rotating the cube through any multiples of $90^{\circ}$ around this axis we effect an interchange of position between four of its sides. This process of interchanging is homologous with rotation through $90^{\circ}$, being in fact equivalent to it, and therefore it is also homologous with multiplication by the imaginary unit. But there is also another homology. Let us designate the four sides of the cube parallel to the axis of rotation as $A, B, C, D$. Then our group of rotations will be homologous with the powers of a cyclic substitution between the four letters $A, B, C, D$.

Let us next introduce a new operator, namely, rotation around an axis at right angles to the first one, but always

NO. I 266 , vOL. 49] 
through an arc of $90^{\circ}$. This introduces a new element into the problem, and enables us to change the cube from any one position to any other position, that is, to effect any interchange among the sides which would be consistent with their remaining sides of the same cube. Here we have a series of rotations which, in the case of the cube, are homologous with certain linear transformations which have been developed by Klein in his very beautiful book on the Icosahedron.

But it is also obvious that in introducing these rotations we are practically operating with quaternions, the operator being a unit vector. Thus we have a homology between certain forms of quaternion multiplication and linear transformations involving the imaginary unit. Moreover, since these rotations are also homologous with substitutions, performed on six symbols representing the six sides of the cube, it follows that there is also a homology between certain groups of substitutions and certain linear transformations involving two quantities, a numerator and a denominator, and quaternion multiplication by unit vectors.

I have taken a cube as the simplest illustration. Evidently we can construct a great number of groups of substitutions of the same sort between the sides of any regular solid, as Klein has done in the work I have already cited. The relation be tween the linear substitutions thus found and the solution of corresponding algebraic equations forms one of the most beautiful branches of our modern mathematics.

We have in all these cases a very simple illustration of a law of thought, the application of which forms the basis of an im portant part of modern mathematical research. We may call it the law of homology. I am not sure of my ability to define it rigorously, but I think we may express it in some such form as this : If we have two sets of concepts, say A and B, such that to every concept of the one set shall correspond a concept of the other, and to every relation between any two of one set a cor responding relation between the corresponding two of the other, then all language, reasoning, and conclusions as to the one set may be applied to the other set. We may, of course, extend the law to a correspondence between things or concepts, and symbols, or other forms of language.

This law is, I think, more universal than might at first sight appear. Not only the progress, but the very existence of our race depends upon that coordination between our mental processes and the processes of the external universe, which has gradually been brought about by the attrition between man and nature through unnumbered generations. A man is perfect, powerful, and effective in proportion as his thoughts of nature coincide with the processes of nature herself; each process of nature having its image in his thought, and vice versa. Now, language consists in coordination between words and conceptions. Thus we pass from nature to what corresponds to it in thought, and from thought to what corresponds to it in language, and thus bring about a correspondence between language and nature.

Modern scientific research affords many examples of the application of this law, which would be very marvellous if they were not so familiar. We are so accustomed to the prediction of an eclipse that we see no philosophy in it. And yet might not a very intellectual being from another sphere see something wonderful in the fact that by a process of making symbols with pen and ink upon sheets of paper, and combining them accord. ing to certain simple rules, it is possible to predict with unerring certainty that the shadow of the moon, on a given day and at a given hour and minute, will pass over a cercain place on the earth's surface? Surely the being might ask with surprise how such a result could be attained. Our reply would be simply this: There is a one-to-one correspondence between the symbols which the mathematician makes on his paper, and the laws of motion of the heavenly bodies. His symbols embody the methods of nature itself.

The introduction and application of homologies such as I have pointed out have, perhaps, their greatest value as thoughtsavers. In the field of mathematical thought they bear some resemblance to labour-saving machines in the field of economics. They enable the results of ratiocination to be reached without going through the process of reasoning in the particular case. Much that I have said illustrates this use of the method, but there is yet another case which has been so fruitful as to be worthy of special mention: I mean the general theory of functions of an imaginary variable. We may regard such functions as being in reality representative of a pair of functions of a certain class involving a pair of real variables; but the difficulty of conceiving the various ways in which the two variables might be related, and the results of the changes which they might go through, in such a way as to clearly follow out all possible results, would have rendered their direct study impossible.

But when Gauss and Cauchy conceived the happy idea of representing two such variables, the real and the imaginary one, by the rectangular coordinates of a point in a plane, those relations which before taxed the powers of conception became comparatively simple. Considered as a magnitude, the complex variable, or the sum of a real quantity and a purely imaginary one, the latter being considered as one measured in imaginary units, was represented by the length and position of a straight line drawn from an origin of coordinates to the point whose coordinates were represented by the values of the variable. Such a line, when both length and direction are considered, is now familiarly known as a vector. The conception of the vector would, however, in many cases be laborious. But the vector is completely determined by its terminal point ; to every vector corresponds one and only one terminal point, and to every terminal point one and only one vector. Hence we may make abstraction of the vector entirely, and in thought attend only to the terminal point. Since for every pair of values we assign to our original variables there is one point, and only one, we may in thought make abstraction of both of these variables, and of the vectors which they represent, and consider only the point whose coordinates they are. Thus the continuous variation of the two quantities, how complex soever it may be, is represented by a motion of the point. Now such a motion is very easy to conceive. We may consider it as performing a number of revolutions around some fixed position without the slightest difficulty, whereas to conceive the corresponding variations in the algebraic variables themselves would need considerable mental effort. Thus, and thus alone, has the beautiful theory, first largely developed by Cauchy, and afterward continued by Riemann, been brought to its present state of perfection.

Another example of the principle in question, where the two objections of reasoning are so nearly of a kind that no thought is saved, is afforded by the principle of duality in projective geometry. Here a one-to-one correspondence is established between the mutual relations of points and lines, with the result that in demonstrating any proposition relating to these concepts we at the same time demonstrate a correlative proposition formed from the original one by simply interchanging the words "point" and "line."

The subjects of which I have heretofore spoken belong conjointly to algebra and geometry. Indeed, one of the great results of bringing homologous interpretation into modern mathematics has been to unify the treatment of algebra and geometry, and almost fuse them into a single science. To a large class of theorems of algebra belong corresponding theo. rems of geometry, each of one class proving one of the other class. Thus the two sciences become mutually helpful. In geometry we have a visible representation of algebraic theorems; by algebraic operations we reach geometrical conclusions which it might be much more difficult to reach by direct reasoning. A remarkable example is afforded by the geometrical application of the theory of invariants. These are perhaps the last kind of algebraic conclusion which the student, when they are first presented to his attention, would conceive to have a geometrical application, yet a very little study suffices to establish a complete homology between them and the distribution of points upon a straight line.

This use of homologies does not mark the only line by which we have advanced beyond our predecessors. Progress has been possible only by emancipating ourselves from certain of the conceptions of ancient geometry which are still uppermost in all our elementary teaching. The illustration I have already given is here much to the point. The expression of a relation between two straight lines by the multiplier which would change one into the other is now familiar to every schoolboy, and the relation itself was familiar to Euclid. But the yet simpler relation of a line to another of equal length standing at right angles to it, and the corresponding operator which will change one into the other, was never thought of by Euclid, and is unfamiliar in our schools. Why is this? It seems to me that it grows out of the ancestral idea that mathematics concerns itself with measurement and that the object of measurement is to express all magnitudes in one-dimensional measure. So completely has 
this idea directed language, that we still extend the use of the word "equal" to all cases of this particular kind of linear equality : we say that a circle is equal to the rectangle contained by its radius and half its circumference. We have therefore been obliged to invent the word "congruent" for absolute equality in all points, or to qualify the adjective "equal " by "identical," saying "identically equal." There is of course no objection to the comparison of magnitudes in this way by reference to one dimensional measures, or by presupposing that the change which one magnitude must undergo in order to be transformed into the other is to be expressed by a single parameter, but changes involving two or any number of parameters, are just as important as those involving one, and the attempt to express all metric relations by referring them to a single parameter has placed such restrictions on thought that it seems to me appropriate to apply the term emancipation to our act in freeing ourselves from them. With us mathematics is no longer the science of quantity. But even if we consider that the ultimate object of mathe. matics is relations between quantities, we have reaped a rich reward by the emancipation, for we are enabled by the use of our broader ideas to reach new conclusions as to metric relations.

The idea of groups of operations, as I have tried to develop it, has in recent years been so extended as to cover a large part of the fields of algebra and geometry. Among the leaders in this extension has been Sophus Lie. Considered from the alge. braic point of view, his idea in its simplest form may be expressed thus: We have a certain quantity, say $x$. We have also an operation of any sort which we may perform upon this quantity. Let this operation depend on a certain quantity, $a$, which necessarily enters into it. As one of the simplest possible examples, we may consider the operation to be that of adding $a$ to $x$. As the quantity $a$ may take an infinity of values, it follows that there will be an infinity of operations all belonging to one class, which operations will be distinguished by the particular value of $a$ in each case. We thus operate on $x$ with one of these operators, and get a certain result, say $x^{\prime}$. We operate on $x^{\prime}$ with a second operator, of the same class, and get a second result, say $x^{\prime \prime}$. If whatever operators we choose from the class, the result $x^{\prime \prime}$ could have been obtained from the original quantity $x$ by some operation of the class, then these operations are such that the product of any two is equivalent to the performance of some one of them. Thus, by repeating them for ever, we could get no results except such as could be obtained by some one operator. To illustrate by one simple example : if our operation consists in the addition of an arbitrary quantity to $x$, then we change $x$ into $x^{\prime}$ by adding a certain quantity $a$ and $x^{\prime}$ into $x^{\prime \prime}$ by adding, a second quantity $b$. The result of these two additions is the same as if we had added in the first place the quantity $a+b$. It need hardly be said that the mul. tiplication by $x$ of any quantity would be another example of the same kind. The pertormance of any number of successive multiplications on a quantity is always equal to a single multiplication by the product of all the factors of the separate multiplications.

These operations are not confined to single quantities. We may consider the operation to be performed upon a system of quantities, which are thus transformed into an equal number of different quantities, each of these new quantities corresponding to one of the first system. If a repetition of the operation upon the second system of quantities gives rise to a third system, which could have been formed from the first system by an operation of the same class, then all these possible operations form a group.

The idea of such systems of operations is by no means new. It has always been obvious, since the general theory of algebraic operations has been studied, that any combination of the operations of addition, multiplication, and division could always be reduced to a system in which there would be only a single operation of division necessary-just as in arithmetic a complex fraction, no' matter what the order of complexity of its terms, can always be reduced to a single simple fraction, that is, to a ratio of two integers, but cannot, in general, be reduced to an integer. Abel made use of this theorem in his celebrated Memoir on the impossibility of solving the general equation of the filth degree.

Another field of maihematical thought, quite distinct from that at which we have just glanced, may be called the fairyland of geometry. To make a mathematician, we must have a higher development of his special power than falls to the lot of other men. When he enters fairyland he must, to do himself justice, take wings which will carry him far above the flights, and even above the sight, of ordinary mortals. To the most imaginative of the latter, a being enclosed in a sphere, the surface of which was absolutely impenetrable, would be so securely imprisoned that not even a spirit could escape except by being so ethereal that it could pass through the substance of the sphere. But the mathematical spirit, in four-dimensional space, could step out without even touching any part of the globe. Taking his stand at a short distance from the earth, he could with his telescope scan every particle of it, from centre to surface, without any necessity that the light should pass through any part of the substance of the earth. If a practised gymnast, he could turn a somersault and come down right side left, just as he looks to our eyes when seen by reflection in a mirror, and that without suffering any distortion or injury whatever. A straight line, or a line which to all our examination would appear straight, if followed far enough, might return into itself. Space itself may have a boundary, or, rather, there may be only a certain quantity of it ; go on for ever, and we would find ourselves always coming back to the starting-point. All these results, too, are reached not merely by facetious forms, but by rigorous geometrical demonstration.

The considerations which lead to the study of these forms of space are so simple that they can be traced without difficulty. When the youth begins the study of plane geometry his attention is devoted entirely to figures lying in a plane. For him space has only two dimensions. To a given point on a straight line only one perpendicular can be drawn. By moving a line of any sort in the plane he can describe a surface, but a solid is wholly without his field. He cannot draw a line from the outside to the inside of a circle without intersecting it. On a given base only two triangles with given sides can be erected, one being on one side of the base, the other on the other. When he reaches solid geometry his conceptions are greatly extended. He can draw any number of perpendiculars to the same point of a straight line. If he has two straight lines perpendicular to each other, he can draw a third straight line which shall be perpendicular to both. A plane surface is not confined to its own plane, but can be moved up and down in such a way as to describe a solid. The characteristic of this motion is that it constantly carries every part of the plane to a position which no part occupied before.

Now, it is a fundamental principle of pure science that the liberty of making hypotheses is unlimited. It is not necessary that we shall prove the hypothesis to be a reality before we are allowed to make it. It is legitimate to anticipate all the possibilities. It is, therefore, a perfectly legitimate exercise of thought to imagine what would result if we should not stop at three dimensions in geometry, but construct one for space having four. As the boy, at a certain stage in his studies, passes from two to three dimensions, so may the mathematician pass from three to four dimensions with equal facility. He does indeed meet with the obstacle that he cannot draw figures in four dimensions, and his faculties are so limited that he cannot construct in his own mind an image of things as they would look in space of four dimensions. But this need not prevent his reasoning on the subject, and one of the most obvious conclusions he would reach is this: As in space of two dimensions one line can be drawn perpendicular to another at a given point, and by adding another dimension to space a third line can be drawn perpendicular to these two; so in a fourth dimension we can draw a line which shall be perpendicular to all three. True, we cannot imagine how the line would look, or where it would be placed, but this is merely because of the limitations of our faculties. As a surface describes a solid by continually leaving the space in which it lies at the moment, so a four-dimensional solid will be generated by a three-dimensional one by a continuous motion which shal constantly be directed outside of this three-dimensional space in which our universe appears to exist. As the man confined in a circle can evade it by stepping over it, so the mathematician, if placed inside a sphere in four-dimensional space, would simply step over it as easily as we should over a circle drawn on the floor. Add a fourth dimension to space, and there is room for an indefinite number of universes, all alongside of each other, as there is for an indefinite number of sheets of paper when we pile them upon each other.

From this point of view of physical science, the question whether the actuality of a fourth dimension can be considered admissible is a very interesting one. All we can say is that,

$$
\text { NO. [ } 266 \text {, VOL } 49 \text { ] }
$$


so far as observation goes, all legitimate conclusions seem to be against it. No induction of physical science is more universal or complete than that three conditions fix the position of a point. The phenomena of light shows that no vibrations go outside of three-dimensional space, even in the luminiferous ether. If there is another universe, or a great number of other universes, outside of our own, we can only say that we have no evidence of their exerting any action upon our own. True, those who are fond of explaining anomalous occurrences, by the action of beings that we otherwise know nothing about, have here a very easy field for their imagination. The question of the sufficiency of the laws of nature to account for all phenomena is, however, too wide a one to be discussed at present.

As illustrating the limitation of our faculties in this direction, it is remarkable that we are unable to conceive of a space of two dimensions otherwise than as contained in one of three. A mere plane, with nothing on each side of it, is to us inconceivable. We are thus compelled, so far as our conceptions go, to accept three dimensions and no more. We have in this a legitimate result of the universal experience through all generations being that of a triply extended space.

Intimately associated with this is the concept of what is sometimes called curved space. I confess that I do not like this expression, as I do not see how space itself can be regarded as curved. Geometry is not the science of space, but the science of figures in space, possessing the properties of extension and mobility which we find to be common to all material bodies. The question raised here is a very old one, and in a general way its history is familiar.

Mathematicians have often attempted to construct geometry without the use of what is commonly called the ninth axiom of Euclid, which seems to me best expressed by saying that in a plane only one line can be drawn which shall be parallel to another line in the plane in the sense of never meeting it in either direction. Yet every attempt to construct an elementary geometry without this axiom has been proved to involve a fallacy in some point of the reasoning. This consideration led Lobatchewsky; and independently of him, I believe, Gauss, to inquire whether a geometry might not be constructed in which this axiom did not hold; in which, in fact, it was possible that if we had two parallel lines in a plane, one of them might turn through a very minute angle without thereby meeting the other line in either direction. The possibility of this was soon shown, and a system of geometry was thus constructed in which the sum of the angles of a plane triangle might be less than two right angles.

Afterward the opposite hypothesis was also introduced. It was found that, given two parallel lines in a plane, it might be supposed that they would ultimately meet in both directions. This hypothesis might even be made without there being more than one point of intersection, each straight line returning into itself. The geometry arising from these two hypotheses has been reduced to a rigorous system by Klein.

To guess the future of mathematical science would be a rash attempt. If made it might seem that, in view of what has been accomplished during our time, the safest course would be to predict great discoveries in this and all other branches of science. The question is sometimes asked whether a mathematical method may not yet be invented which shall be as great an advance on the infinitesimal calculus as the latter was on the methods of Euclid and Diaphuntus. So far as solving problems which now confront us is concerned, I am not sure that the safest course would not be to answer such questions in the negative. Is it not true in physics as in mathematics that great discoveries have been made on unexpected lines, and that the pronlems which perplexed our ancestors now baffle our own efforts? We must also remember that the discovery of what could not be done has been an important element in progress. We are met at every step by the iron law of the conservation of energy: in every direction we see the limits of the possible. The mathematics of the twenty-first century may be very different from our own; perhaps the schoolboy will begin algebra with the theory of substitution-groups, as he might now but for inherited habits. But we may well doubt whether our posterity will solve many problems which we cannot, or invent an algorithm more powerful than the calculus. The first principles of all our mathemaical methods are as old as Euclid, and we cannot ex pect that the future will do more than apply them to new problems.

$$
\text { No. I } 266 \text {, vo[. } 49 \text { ] }
$$

\section{UNIVERSITY AND EDUCATIONAL INTELLIGENCE.}

OXFORD. - At a meeting of the Junior Scientific Club, held on Friday, January $26, \mathrm{Mr}$. Pycraft exhibited a restoration of the wing of archæopteryx; Mr. F. A. Hillard read a paper on carborundum, and other substances, prepared by means of the electric arc; and Mr. H. M. Vernon read a paper on the activity of the cardiac centre under varying conditions. It was agreed at the meeting that the annual Boyle lecture, which will be given this year by Prof. Macalister, should be held early in May, and that a conversazione should be given on another day in the summer term.

CAMbridge.-Mr. J. W. Capstick, Fellow of Trinity, has been appointed an Assistant Demonstrator in Physics at the Cavendish Laboratory, in the room of Mr. Whetham, who has been elected to the Clerk Maxwell Scholarship.

A course of lectures with demonstrations in elementary physiology for students of agriculture will be given this term by Mr. Eichbolz, Fellow of Emmanuel College, on Mondays and Saturdays, at nine.

Baron Anatole von Hiigel, Curator of the Museum of Archæology and Ethnology, will this term give two courses of lectures on the collections in the Museum.

A syndicate, consisting of the Vice-Chancellor, the Master of Peterhouse, the Master of Christ's, Prof. Thomson, F.R.S., Prof. Liveing, F.R.S., Mr. Glazebrook, F.R.S., and Mr. Shaw, F.R.S., is about to be appointed to consider the best means of extending the Cavendish Laboratory. A site for the extension is reserved, but the standing difficulty of funds is likely to prove a serious one unless outside help can be obtained.

Dr. P. W. Latham has resigned the Downing Professorship of Medicine, which he has held since 1874. The appointment is made by a special board of electors. Mr. J. R. Green, of Trinity College, Professor of Botany to the Royal Pharmaceutical Society of Great Britain, has been approved by the General Board of Studies for the degree of !) octor of Science.

It is proposed to admit to the privileges of affiliated students, matriculated members of the University of Adelatde who have studied there for two years in arts, law, science, or medicine, and have passed certain specified examinations. Such affiliated students are exempted from the Previous Examination and from one year of residence for the B.A. degree.

\section{SCIENTIFIC SERIALS.}

American Meteorological Journal, January.-History of the Weather Map, by M. W. Harrington. Simultaneous observ. ations, which form the basis of weather charts, were made in Virginia from 1772 to 1777 ; about the same time Lavoisier proposed that such observations should be made in Europe, and referred to an earlier proposal by Borda. In 1842, Kreil, of Prague, proposed the use of an electromagnetic telegraph for the same purpose. The earliest proposal for a weather map was probably made by Brandes, in 1816 , but his plan seems never to have been carried out, and it was not until 1856 that current charts of the weather were made by the Smithsonian Institution. In 1857 , Le Verrier published an international bulletin, but his synoptic charts were not issued until 1863 ; and in this country Admiral FitzRoy commenced the publication of tele. graphic weather reports in 1860 ; since this time such reports and charts became general. - The meteorological work of the Medical Department of the United States Army, by Major C. Smart. The earliest meteorological journal in the office of the Surgeon-General is from Cambridge, for July, 1816 . The first results were published in the Meteorological Fegister for the years 1822-5. - The meteorological work of the Smithsonian Institution, by S. P. Langley. In December, 1847, Prof. J. Henry proposed a "system of extended meteorological observations for solving the problem of American storms," and shortly afterwards the institution issued directions for meteorological observations; in 1849 elementary telegraphic weather reports were furnished to the institution daily.-Early individual observers in the United States, by A. J. Henry. A daily record of the weather was kept by the Rev. J. Campanius at Fort Christiania, near the present city of Wilmington, Delaware, during $1644-5$, and at Boston, by the Hon. P. Dudley, in 